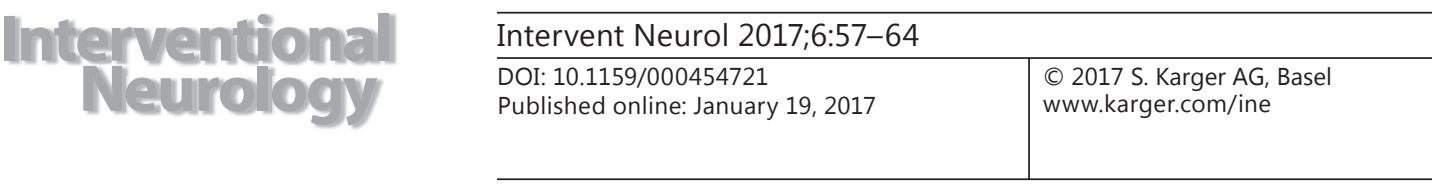

\title{
Risk of Intracranial Hemorrhage after Endovascular Treatment for Acute Ischemic Stroke: Systematic Review and Meta-Analysis
}

\author{
Yonggang Hao ${ }^{a, c}$ Zhizhong Zhang ${ }^{b}$ Hao Zhang $^{b}$ Lili Xu ${ }^{b}$ Zusen Ye ${ }^{a}$ \\ Qiliang Daib Xinfeng Liu ${ }^{a}$ Gelin Xua \\ a Department of Neurology, Jinling Hospital, Southern Medical University, and \\ b Department of Neurology, Jinling Hospital, Medical School of Nanjing University, Nanjing, \\ and ${ }^{\mathrm{C}}$ Department of Emergency Medicine, First Affiliated Hospital of Soochow University, \\ Suzhou, China
}

\section{Keywords}

Endovascular treatment $\cdot$ Intracranial hemorrhage $\cdot$ Recanalization $\cdot$ Stroke

\begin{abstract}
Background: Intracranial hemorrhage is a major complication of endovascular treatment in patients with acute ischemic stroke. Controlled clinical trials reported varied incidences of intracranial hemorrhage after endovascular treatment. This meta-analysis aimed to estimate whether endovascular treatment, compared with medical treatment, increases the risk of intracranial hemorrhage in patients with acute ischemic stroke. Methods: The current publications on endovascular treatment for acute ischemic stroke were systematically reviewed. Rates of intracranial hemorrhage after endovascular treatment for acute ischemic stroke reported in controlled clinical trials were pooled and analyzed. Random and fixed-effect models were used to pool the outcomes. For analyzing their individual risks, intracranial hemorrhages after endovascular treatment were classified as symptomatic and asymptomatic. Results: Eleven studies involving 1,499 patients with endovascular treatment and 1,320 patients with medical treatment were included. After pooling the data, the risk of any intracranial hemorrhage was significantly higher in patients with endovascular treatment than in patients with medical treatment (35.0 vs. $19.0 \%, \mathrm{OR}=2.55,95 \%$ CI: $1.64-3.97, p<0.00001)$. The risk of asymptomatic intracranial hemorrhage was also significantly higher in patients with endovascular treatment than in those with medical treatment ( 28 vs. $12 \%$, OR $=3.16,95 \% \mathrm{CI}: 1.62-6.16, p<$ 0.001 ). However, the risks of symptomatic intracranial hemorrhage were similar in patients
\end{abstract}

Yonggang Hao and Zhizhong Zhang contributed equally to this work.

Prof. Gelin Xu

Department of Neurology, Jingling Hospital, Southern Medical University

305\# East Zhongshan Road

Nanjing, Jiangsu 210002 (China)

E-Mail gelinxu@ nju.edu.cn 
with endovascular treatment and in those with medical treatment (5.6 vs. $5.2 \%, \mathrm{OR}=1.09$, $95 \%$ CI: $0.79-1.50, p=0.61)$. Conclusion: Although the risk of any intracranial hemorrhage may increase after endovascular treatment, the risk of symptomatic intracranial hemorrhage may remain similar as compared with medical treatment.

(C) 2017 S. Karger AG, Basel

\section{Introduction}

As a major complication of endovascular treatment in patients with acute ischemic stroke, intracranial hemorrhage may neutralize or even invert the benefit-risk ratio of the treatments [1]. Several carefully designed clinical trials have validated the efficacy of endovascular treatment over medical treatment in patients with acute ischemic stroke, but these trials reported varied rates of intracranial hemorrhage [2-7]. The higher incidences of intracranial hemorrhage have been attributed to cases with asymptomatic intracranial hemorrhage after endovascular treatment. Given the limited cases with intracranial hemorrhage in individual studies, this interpretation may be less convincing. Although the efficacy of endovascular treatment over medical treatment has been reviewed in recent meta-analyses [8-10], the risk of intracranial hemorrhage after these 2 treatment strategies has not been systematically evaluated.

After the endovascular treatment recommended by the AHA/ASA guidelines for acute ischemic stroke [11], the number of patients treated with this new strategy is expected to increase considerably. Therefore, evaluating the risk of intracranial hemorrhage becomes an important issue for continuously improving the efficacy of this new treatment strategy. Based on the current evidence, this meta-analysis assessed whether endovascular treatment, compared with medical treatment, increases the risk of intracranial hemorrhage in patients with acute ischemic stroke.

\section{Methods}

\section{Search Strategy and Selection Criteria}

This meta-analysis follows the guidelines introduced by the Preferred Reporting Items for Systematic Reviews and Meta-Analyses (PRISMA) statement. We searched PubMed, Web of Science, and Cochrane Library for publications in English from 1995 to 2015. The following search formula was used: (ischemic stroke OR cerebral infarct OR brain infarct) AND (intra-arterial thrombolysis OR intra-arterial therapy OR endovascular therapy OR endovascular treatment OR interventional therapy OR thrombectomy OR embolectomy).

All returned publications were evaluated by 2 authors (Z.Y. and H.Z.) independently. Studies meeting all following criteria were included: controlled clinical trials comparing endovascular treatment with medical treatment in patients with acute ischemic stroke; at least 20 patients enrolled for endovascular approach; involved functional follow-up of 3 months or longer, and reported incidences of symptomatic intracranial hemorrhage. Animal and experimental studies were not included. Data of multiple publications from 1 study were combined. Considering the less application in current clinical practice, trials with urokinase as the main thrombolytic agent were excluded.

Data Extraction and Quality Assessment

Two authors (Y.H. and H.Z.) extracted data from the included studies independently. Any disagreements were resolved by discussion. The primary outcome was the rates of symptomatic intracranial hemorrhage. The secondary outcomes included any intracranial hemorrhage, asymptomatic intracranial hemorrhage, parenchymal hemorrhage, and subarachnoid hemorrhage (SAH). The methodological quality of randomized controlled trials (RCTs) was assessed with the Cochrane risk of bias tool [12]. Retrospective studies were assessed with the modified Newcastle-Ottawa scale ( $\geq 6$ indicates high quality) [13]. 
Table 1. Baseline characteristics of the clinical trials included

\begin{tabular}{|c|c|c|c|c|c|c|c|}
\hline Trials (year of publication) & Design & $\begin{array}{l}\text { Treatment in } \\
\text { intervention }\end{array}$ & $\begin{array}{l}\text { Treatment in } \\
\text { controls }\end{array}$ & $\begin{array}{l}\text { Intervention/ } \\
\text { controls, } n\end{array}$ & $\begin{array}{l}\text { Time } \\
\text { window of } \\
\text { ET/IVT, h }\end{array}$ & $\begin{array}{l}\text { Time of } \\
\text { follow-up, } \\
\text { days }\end{array}$ & $\begin{array}{l}\text { Quality } \\
\text { score }\end{array}$ \\
\hline IMS [18], 2004 & $\mathrm{RP}$ & IAT & IVT & $80 / 182$ & $5 / 3$ & 90 & 7 \\
\hline IMS II [19], 2007 & $\mathrm{RP}$ & IAT & IVT & $81 / 182$ & $5 / 3$ & 90 & 7 \\
\hline Synthesis [17], 2010 & PROBE & IAT/ME & IVT & $25 / 29$ & $6 / 3$ & 90 & RCT \\
\hline Synthesis Exp [16], 2013 & PROBE & $\mathrm{IAT} / \mathrm{ME}$ & IVT & $181 / 181$ & $6 / 4.5$ & 90 & RCT \\
\hline IMS III [15], 2013 & PROBE & IAT/ME/IVT & IVT & $434 / 222$ & $5 / 3$ & 90 & RCT \\
\hline MR-RESCUE [14], 2013 & PROBE & IAT/ME/IVT & IVT/BM & $64 / 54$ & $8 / 4.5$ & 90 & RCT \\
\hline MR CLEAN [2], 2015 & PROBE & IAT/ME/IVT & IVT/BM & $233 / 267$ & $6 / 4.5$ & 90 & RCT \\
\hline Escape [4], 2015 & PROBE & $\mathrm{ME} / \mathrm{IVT}$ & IVT/BM & $120 / 118$ & $12 / 4.5$ & 90 & $\mathrm{RCT}$ \\
\hline EXTEND-IA [5], 2015 & PROBE & ME/IVT & IVT & $35 / 35$ & $6 / 4.5$ & 90 & $\mathrm{RCT}$ \\
\hline SWIFT PRIME [6], 2015 & PROBE & ME/IVT & IVT & 98/98 & $6 / 4.5$ & 90 & RCT \\
\hline Revascat [3], 2015 & PROBE & ME/IVT & IVT/BM & $103 / 103$ & $8 / 4.5$ & 90 & RCT \\
\hline
\end{tabular}

$\mathrm{RP}$, retrospective design, prospective data collection; PROBE, prospective randomized open-label blinded-endpoint design; ET, endovascular treatments; IAT, intra-arterial thrombolysis; ME, mechanical embolectomy; IVT, intravenous t-PA only; BM, best medical management; RCT, randomized controlled trail.

Statistical Analysis and Evidence Synthesis

Statistical analyses were performed using the Review Manager 5.3 (Cochrane Collaborane Collaboration, Oxford, UK) and Stata 12.0 (Stata Corp LP, College Station, TX, USA) software. The odds ratio (OR) and associated $95 \%$ confidence interval (CI) were used to compare dichotomous variables. Heterogeneity among studies was assessed with the Cochran Q test and the Higgins $I^{2}$ test. A Cochran's Q of $p<0.10$ and $I^{2}>50 \%$ were considered as significant heterogeneity. The fixed-effect model was used if there was no significant heterogeneity among studies. Otherwise, the random-effect model was used. Sensitivity analyses were implemented for high-quality studies. The funnel plots, Egger test, and Begg test were used to evaluate publication bias. Meta-regression analysis was conducted to explore the potential for sources of heterogeneity.

\section{Results}

Eleven studies meeting the inclusion criteria were identified (online suppl. Fig. 1; for all online suppl. material, see www.karger.com/doi/10.1159/000454721), which involved 1,499 patients with endovascular treatment and 1,320 patients with medical treatment. The characteristics of the included studies are shown in Table 1 . There were 9 RCTs $[2-6,14-17]$ and 2 single-arm studies $[18,19]$ which used historical series as controls. The reported endovascular procedures included intra-arterial thrombolysis, balloon angioplasty, stent implantation, and mechanical embolectomy with different devices. Some endovascular treatments were bridged with intravenous thrombolysis, others were not. The 2 arms, with and without ischemic penumbra, stratified in the endovascular treatment group in the Mechanical Retrieval and Recanalization of Stroke Clots Using Embolectomy (MR-RESCUE) trial were combined for data analysis [14]. Since the same patient series with intravenous thrombolysis was used as historical control in the Interventional Management of Stroke (IMS) I and II studies $[18,19]$, these patients were counted only once when pooling the sample size. These studies were estimated for risks of bias. All enrolled RCTs were identified to have a high risk for performance bias as the patients and stroke teams could not be blinded to treatment allocations, which may generate biases during outcome reporting. 


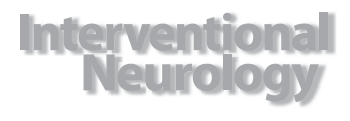

\begin{tabular}{l|l}
\hline \multicolumn{2}{l}{ Intervent Neurol 2017;6:57-64 } \\
\hline DOI: 10.1159/000454721 & $\begin{array}{l}\text { C 2017 S. Karger AG, Basel } \\
\text { www.karger.com/ine }\end{array}$ \\
\hline
\end{tabular}

Hao et al.: Risk of Intracranial Hemorrhage after Endovascular Treatment for Acute

Ischemic Stroke: Systematic Review and Meta-Analysis

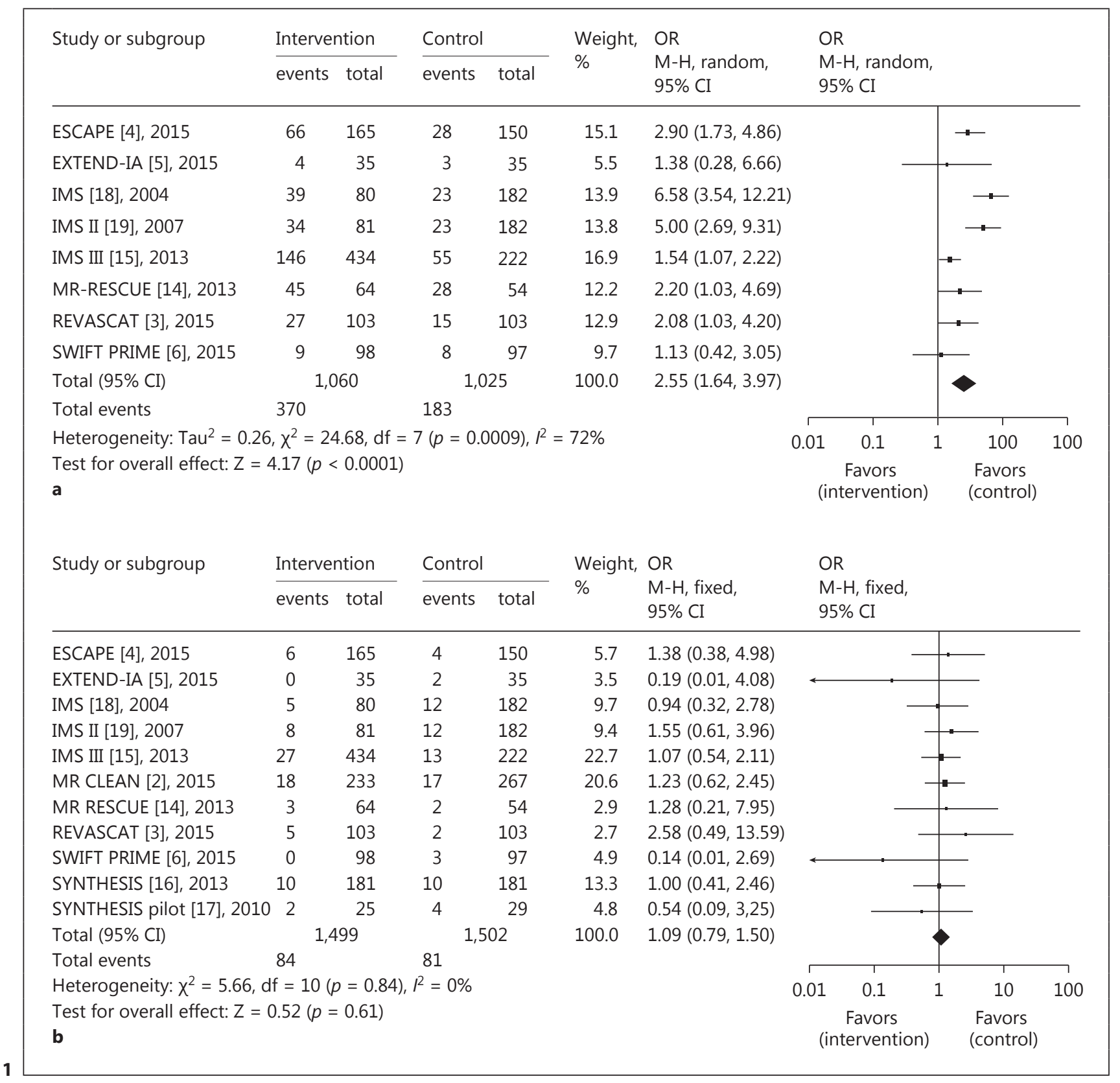

(Figure continued on next page.)

Eight of the 11 enrolled studies reported rates of any intracranial hemorrhage. After pooling these data, the risk of any intracranial hemorrhage was significantly higher in patients with endovascular treatment than in patients with medical treatment $(35.0$ vs. $19.0 \%, 0 \mathrm{R}=$ 2.55, 95\% CI: 1.64-3.97, $p<0.00001)$, with a high heterogeneity among studies $\left(I^{2}=72 \%\right.$, $p<0.001$; Fig. 1a). After excluding 2 nonrandomized control trials (IMS I and IMS II), the risk of any intracranial hemorrhage was still significantly higher in the endovascular arm (OR = 1.88, 95\% CI: $1.47-2.40, p<0.00001$ ), with a relatively low heterogeneity among studies $\left(I^{2}=6 \%, p=0.38\right)$.

All 11 enrolled studies reported rates of symptomatic intracranial hemorrhage. The risk of symptomatic intracranial hemorrhage was much similar in patients treated with endovas- 
Hao et al.: Risk of Intracranial Hemorrhage after Endovascular Treatment for Acute

Ischemic Stroke: Systematic Review and Meta-Analysis

\begin{tabular}{|c|c|c|c|c|c|c|c|c|c|c|c|}
\hline \multirow{3}{*}{$\begin{array}{l}\text { Study or subgroup } \\
\text { EXTEND-IA [5], } 2015\end{array}$} & \multicolumn{2}{|c|}{ Intervention } & \multicolumn{2}{|l|}{ Control } & \multirow{2}{*}{$\begin{array}{l}\text { Weight, } \\
\%\end{array}$} & \multirow{2}{*}{$\begin{array}{l}\text { OR } \\
\mathrm{M}-\mathrm{H}, \text { random, } \\
95 \% \mathrm{CI}\end{array}$} & \multirow{2}{*}{\multicolumn{3}{|c|}{$\begin{array}{l}\text { OR } \\
\text { M-H, random, } \\
95 \% \text { CI }\end{array}$}} & & \\
\hline & events & total & events & total & & & & & & & \\
\hline & 4 & 35 & 1 & 35 & 6.1 & $4.39(0.46,41.40)$ & & & & & \\
\hline IMS [18], 2004 & 34 & 80 & 11 & 182 & 15.8 & $11.49(5.41,24.42)$ & & & & $\longrightarrow$ & \\
\hline IMS II [19], 2007 & 26 & 81 & 11 & 182 & 15.7 & $7.35(3.41,15.83)$ & & & & $\longrightarrow$ & \\
\hline IMS III [15], 2013 & 119 & 434 & 42 & 222 & 18.5 & $1.62(1.09,2.41)$ & & & & - & \\
\hline MR RESCUE [14], 2013 & 42 & 64 & 26 & 54 & 15.9 & $2.06(0.98,4.32)$ & & & & - & \\
\hline REVASCAT [3], 2015 & 17 & 103 & 11 & 103 & 15.3 & $1.65(0.73,3.73)$ & & & & & \\
\hline SWIFT PRIME [6], 2015 & 9 & 98 & 5 & 97 & 12.6 & $1.86(0.60,5.77)$ & & & & & \\
\hline Total $(95 \% \mathrm{CI})$ & \multicolumn{2}{|c|}{895} & \multicolumn{2}{|c|}{875} & 100.0 & $3.16(1.62,6.16)$ & & & & & \\
\hline Total events & \multicolumn{2}{|c|}{251} & \multicolumn{2}{|c|}{107} & & & \multirow{3}{*}{0.01} & 1 & & 1 & $\longrightarrow$ \\
\hline \multirow{2}{*}{\multicolumn{7}{|c|}{$\begin{array}{l}\text { Heterogeneity: } \text { Tau }^{2}=0.59, \chi^{2}=29.56, d f=6(p<0.0001), I^{2}=80 \% \\
\text { Test for overall effect: } Z=3.36(p=0.0008) \\
\text { c }\end{array}$}} & & 0.1 & 1 & 10 & 100 \\
\hline & & & & & & & & \multicolumn{3}{|c|}{$\begin{array}{c}\text { Favors } \\
\text { (intervention) }\end{array}$} & \\
\hline \multirow[t]{2}{*}{ Study or subgroup } & \multicolumn{2}{|c|}{ Intervention } & \multicolumn{2}{|l|}{ Control } & \multirow{2}{*}{$\begin{array}{l}\text { Weight, } \\
\%\end{array}$} & \multirow{2}{*}{$\begin{array}{l}\text { OR } \\
\text { M-H, fixed, } \\
95 \% \mathrm{CI}\end{array}$} & \multirow{2}{*}{\multicolumn{3}{|c|}{$\begin{array}{l}\text { OR } \\
\text { M-H, fixed, } \\
95 \% \text { CI }\end{array}$}} & & \\
\hline & events & total & events & total & & & & & & & \\
\hline IMS III [15], 2013 & 48 & 417 & 12 & 207 & 81.0 & $2.11(1.10,4.07)$ & & & & & \\
\hline MR CLEAN [2], 2015 & 2 & 233 & 0 & 267 & 2.6 & $5.78(0.28,120.96)$ & & & & & $\rightarrow$ \\
\hline REVASCAT [3], 2015 & 5 & 103 & 2 & 103 & 10.9 & $2.58(0.49,13.59)$ & & & & & \\
\hline SWIFT PRIME [6], 2015 & 4 & 98 & 1 & 97 & 5.5 & $4.09(0.45,37.23)$ & & & & & \\
\hline Total $(95 \%$ CI) & 85 & 1 & & 4 & 100.0 & $2.37(1.33,4.22)$ & & & & & \\
\hline Total events & 59 & & 15 & & & & & 1 & & 1 & $\square$ \\
\hline \multicolumn{7}{|c|}{ Heterogeneity: $\chi^{2}=0.69, \mathrm{df}=3(p=0.88), R^{2}=0 \%$} & 0.01 & 0.1 & 1 & 10 & 100 \\
\hline \multicolumn{5}{|c|}{$\begin{array}{l}\text { Test for overall effect: } Z=2.93(p=0.003) \\
\text { d }\end{array}$} & & & \multicolumn{3}{|c|}{$\begin{array}{c}\text { Favors } \\
\text { (intervention) }\end{array}$} & $\begin{array}{l}\text { Favors } \\
\text { (control) }\end{array}$ & \\
\hline
\end{tabular}

Fig. 1. Forest plot of hazard ratio by patient subgroups.

cular procedures and with medicines (5.6 vs. $5.2 \%, \mathrm{OR}=1.09,95 \% \mathrm{CI}: 0.79-1.50, p=0.61$; Fig. $1 b)$, with a very low heterogeneity among studies $\left(I^{2}=0 \%, p=0.89\right)$. Seven of the 11 enrolled studies provided valid data of asymptomatic intracranial hemorrhage. The risk of asymptomatic intracranial hemorrhage was significantly higher in patients with endovascular treatment than in patients with medical treatment $(28.0$ vs. $13.9 \%, \mathrm{OR}=3.16,95 \% \mathrm{CI}$ : $1.62-6.16, p<0.001$; Fig. 1c), with a relatively high heterogeneity among studies $\left(I^{2}=80 \%\right.$, $p<0.0001$ ). After excluding 2 nonrandomized control trials (IMS I and IMS II), the risk of asymptomatic intracranial hemorrhage was still significantly higher in the endovascular arm (OR $=1.74,95 \% \mathrm{CI}: 1.28-2.37, p<0.001)$, but the heterogeneity among studies decreased considerably $\left(I^{2}=0, p=0.91\right)$.

Seven of the 11 enrolled studies reported rates of parenchymal hemorrhage. When the data were pooled, no significant differences concerning the risk of parenchymal hemorrhage were detected between patients with endovascular treatment and those with medical treatment ( 7.7 vs. $5.7 \%, \mathrm{OR}=1.23,95 \% \mathrm{CI}$ : $0.86-1.75, p=0.25$; online suppl. Fig. 2 ). Four studies provided valid data on SAH. The reported SAH incidence was higher in patients with endovascular treatment than in patients with medical treatment ( 6.9 vs. $2.2 \%$, $O R=2.37,95 \%$ CI: $1.33-4.22, p<0.01$; Fig. 1 d). 
When data from randomized control trails were pooled for sensitivity analysis, there was no change concerning the difference level of the outcomes (online suppl. Table 1). Fixed-effect and random-effect models induced consistent results in sensitivity analyses. Univariate metaregression analyses showed that nonrandomized control trials were more heterogeneous when evaluating the risk of asymptomatic intracranial hemorrhage $(p=0.003)$. No significant publication biases were detected by the funnel plots, the Egger test, and the Begg test when evaluating the risk of symptomatic intracranial hemorrhage (online suppl. Table 2, Fig. 3).

\section{Discussion}

This meta-analysis showed that endovascular treatments increased the risk of any intracranial hemorrhage compared with medical treatment. This increased risk was mainly due to extra cases with asymptomatic intracranial hemorrhage after endovascular treatment. Although the risk of SAH increased in patients with endovascular treatment, the risk of symptomatic hemorrhage remained much similar as compared with that in patients with medical treatment.

During or after endovascular treatment for acute ischemic stroke, intracranial hemorrhage may result from mechanical lesion of the vessel wall, reperfusion lesion, increased blood-brain barrier permeability [20], hemorrhagic tendency related to thrombolysis agents or heparinization [21,22], and hemodynamic lesion due to fluctuation of blood pressure [23]. Intracranial hemorrhage resulting from mechanical lesions of vessels is limited to patients with endovascular treatment, which is not possible in patients with medical treatment. Mechanical penetration of the vessel wall usually occurs during or shortly after the guidewire crosses the target occlusion, a circumstance that arises when the artery is not visualized under X-ray fluoroscopy. Retrieving the stent-like devices may displace the target artery and break small perforate arteries. Most of these occasions may result in SAH or intracerebral hemorrhage. These complications related to endovascular procedures are largely responsible for the increased risk of endovascular treatment [24]. This meta-analysis confirmed that the risk of SAH was increased in patients with endovascular treatment. Since complications related to endovascular procedures are highly skill dependent, the risk of SAH was lower in centers with a large patient volume, in the hands of experienced operators, and in recent studies with more advanced devices $[2,4,5]$.

A reperfusion lesion may occur after the recanalization of the target occluded the artery [25]. Hemorrhage may result from rapture of the necrotic vessel wall and increased bloodbrain barrier permeability due to prolonged ischemia [20]. Therefore, hemorrhage due to a reperfusion lesion is relevant to the degree and tempo of the reperfusion flow, the pretreatment infarct volume, and the time of recanalization. More recent studies reported higher rates of recanalization (58.7-88.0\%), but also higher rates of intracranial hemorrhage (9.2-40.0\%) after endovascular treatment [3-6]. Minor or asymptomatic intracranial hemorrhage in CT has been suggested as an indicator of successful reperfusion.

Imaging examinations may influence the rates of symptomatic intracranial hemorrhage. The incidences of symptomatic intracranial hemorrhage in MR CLEAN [2] and THERAPY [26] were higher than those reported in SWIFT PRIME [6] and EXTEND-IA [5] which excluded patients with large ischemic core. Infarct volume was negatively related to baseline collateral status [27] and time delay from symptoms onset to recanalization [28]. As in the EXTEND-IA trial, CTA and CTP were used to exclude patients with large infarct cores for endovascular treatment. Favorable outcomes were observed in patients with moderate-to-good collateral circulation and in those with small infarct core. The incidence of symptomatic intracranial hemorrhage did not increase despite the time window for endovascular treatment was ex- 
tended to $12 \mathrm{~h}$ after stroke onset $[4,5]$. These results suggested that recanalization treatment initiated even $12 \mathrm{~h}$ after stroke onset may result in favorable outcomes in selected patients.

Several limitations should be emphasized when interpreting the results of this metaanalysis. Some potential confounders which may influence the risk of intracranial hemorrhage were not analyzed in this study. Different thrombectomy devices were used in these trials. The types of endovascular device may influence the risk of intracranial hemorrhage. One study showed that stent-like thrombus retrievers may decrease intracranial hemorrhage compared with previously developed devices [29]. The time window for endovascular treatment varied considerably in the 11 included studies. The incidence of intracranial hemorrhage may increase with the delay of recanalization treatment. Another potential confounder is the varied definitions of symptomatic intracranial hemorrhage [30]. At least 4 different definitions were used in these 11 studies [31-34]. One major concern for these definitions lies in that different time intervals between treatment and CT scan were proposed (online suppl. Table 3). Since small intracranial hemorrhage may be absorbed in time, the longer interval between symptom onset and CT scan may indicate a decreased detection rate. The criteria for symptomatic intracranial hemorrhage were also discrepant, which may lead to different reported incidences of the symptomatic intracranial hemorrhage. The recently published Heidelberg Bleeding Classification may provide a reasonable solution for this issue [35]. Furthermore, some trials were terminated prematurely due to a significantly futile or beneficial effect in interim analysis $[3-6,15]$, which may have attenuated the power when evaluating the risk of hemorrhage.

\section{Summary}

Compared with medical treatment, endovascular treatment may increase the risk of asymptomatic intracranial hemorrhage, but not the risk of symptomatic intracranial hemorrhage in patients with ischemic stroke due to large vessel occlusion. Imaging-based patient selection for endovascular treatment may reduce the risk of symptomatic intracranial hemorrhage after recanalization. Future studies should consider using uniformed definitions and standardized procedures in diagnosing intracranial hemorrhage.

\section{Disclosure Statement}

The authors have no conflicts of interest to disclose.

\section{References}

1 Thanvi BR, Treadwell S, Robinson T: Haemorrhagic transformation in acute ischaemic stroke following thrombolysis therapy: classification, pathogenesis and risk factors. Postgrad Med J 2008;84:361-367.

2 Berkhemer OA, Fransen PS, Beumer D, et al: A randomized trial of intraarterial treatment for acute ischemic stroke. N Engl J Med 2015;372:11-20.

3 Jovin TG, Chamorro A, Cobo E, et al: Thrombectomy within 8 hours after symptom onset in ischemic stroke. N Engl J Med 2015;372:2296-2306.

4 Goyal M, Demchuk AM, Menon BK, et al: Randomized assessment of rapid endovascular treatment of ischemic stroke. N Engl J Med 2015;372:1019-1030.

5 Campbell BC, Mitchell PJ, Kleinig TJ, et al: Endovascular therapy for ischemic stroke with perfusion-imaging selection. N Engl J Med 2015;372:1009-1018.

6 Saver JL, Goyal M, Bonafe A, et al: Stent-retriever thrombectomy after intravenous t-PA versus t-PA alone in stroke. N Engl J Med 2015;372:2285-2295.

7 Paciaroni M, Inzitari D, Agnelli G, et al: Intravenous thrombolysis or endovascular therapy for acute ischemic stroke associated with cervical internal carotid artery occlusion: the ICARO-3 study. J Neurol 2015;262:459-468. 
8 Phan K, Zhao DF, Phan S, et al: Endovascular therapy including thrombectomy for acute ischemic stroke: a systematic review and meta-analysis with trial sequential analysis. J Clin Neurosci 2016;29:38-45.

9 Badhiwala JH, Nassiri F, Alhazzani W, et al: Endovascular thrombectomy for acute ischemic stroke: a metaanalysis. JAMA 2015;314:1832-1843.

10 Goyal M, Menon BK, van Zwam WH, et al: Endovascular thrombectomy after large-vessel ischaemic stroke: a meta-analysis of individual patient data from five randomised trials. Lancet 2016;387:1723-1731.

11 Powers WJ, Derdeyn CP, Biller J, et al: 2015 American Heart Association/American Stroke Association focused update of the 2013 guidelines for the early management of patients with acute ischemic stroke regarding endovascular treatment: a guideline for healthcare professionals from the American Heart Association/American Stroke Association. Stroke 2015;46:3020-3035.

12 Higgins JP, Altman DG, Gotzsche PC, et al: The Cochrane Collaboration's tool for assessing risk of bias in randomised trials. BMJ 2011;343:d5928.

13 Stang A: Critical evaluation of the Newcastle-Ottawa scale for the assessment of the quality of nonrandomized studies in meta-analyses. Eur J Epidemiol 2010;25:603-605.

14 Kidwell CS, Jahan R, Gornbein J, et al: A trial of imaging selection and endovascular treatment for ischemic stroke. N Engl J Med 2013;368:914-923.

15 Broderick JP, Palesch YY, Demchuk AM, et al: Endovascular therapy after intravenous t-PA versus t-PA alone for stroke. N Engl J Med 2013;368:893-903.

16 Ciccone A, Valvassori L, Nichelatti M, et al: Endovascular treatment for acute ischemic stroke. N Engl J Med 2013;368:904-913.

17 Ciccone A, Valvassori L, Ponzio M, et al: Intra-arterial or intravenous thrombolysis for acute ischemic stroke? The SYNTHESIS pilot trial. J Neurointerv Surg 2010;2:74-79.

18 The IMS Study Investigators: Combined intravenous and intra-arterial recanalization for acute ischemic stroke: the Interventional Management of Stroke Study. Stroke 2004;35:904-911.

19 The IMS II Trial Investigators: The Interventional Management of Stroke (IMS) II Study. Stroke 2007;38:21272135.

20 Rosell A, Cuadrado E, Ortega-Aznar A, Hernandez-Guillamon M, Lo EH, Montaner J: MMP-9-positive neutrophil infiltration is associated to blood-brain barrier breakdown and basal lamina type IV collagen degradation during hemorrhagic transformation after human ischemic stroke. Stroke 2008;39:1121-1126.

21 The NINDS t-PA Stroke Study Group: Intracerebral hemorrhage after intravenous t-PA therapy for ischemic stroke. Stroke 1997;28:2109-2118.

22 International Stroke Trial Collaborative Group: The International Stroke Trial (IST): a randomised trial of aspirin, subcutaneous heparin, both, or neither among 19,435 patients with acute ischaemic stroke. Lancet 1997;349:1569-1581.

23 Butcher K, Christensen S, Parsons M, et al: Postthrombolysis blood pressure elevation is associated with hemorrhagic transformation. Stroke 2010;41:72-77.

24 Gill HL, Siracuse JJ, Parrack IK, Huang ZS, Meltzer AJ: Complications of the endovascular management of acute ischemic stroke. Vasc Health Risk Manag 2014;10:675-681.

25 Furlan A, Higashida R, Wechsler L, et al: Intra-arterial prourokinase for acute ischemic stroke. The PROACT II study: a randomized controlled trial. Prolyse in Acute Cerebral Thromboembolism. JAMA 1999;282:20032011.

26 Mocco J, Zaidat 00, von Kummer R, et al: Aspiration thrombectomy after intravenous alteplase versus intravenous alteplase alone. Stroke 2016;47:2331-2338.

27 Bang OY, Saver JL, Kim SJ, et al: Collateral flow averts hemorrhagic transformation after endovascular therapy for acute ischemic stroke. Stroke 2011;42:2235-2239.

28 Molina CA, Montaner J, Abilleira S, et al: Timing of spontaneous recanalization and risk of hemorrhagic transformation in acute cardioembolic stroke. Stroke 2001;32:1079-1084.

29 Saver JL, Jahan R, Levy EI, et al: Solitaire flow restoration device versus the Merci Retriever in patients with acute ischaemic stroke (SWIFT): a randomised, parallel-group, non-inferiority trial. Lancet 2012;380:12411249.

30 Seet RC, Rabinstein AA: Symptomatic intracranial hemorrhage following intravenous thrombolysis for acute ischemic stroke: a critical review of case definitions. Cerebrovasc Dis 2012;34:106-114.

31 The National Institute of Neurological Disorders and Stroke rt-PA Stroke Study Group: Tissue plasminogen activator for acute ischemic stroke. N Engl J Med 1995;333:1581-1587.

32 Hacke W, Kaste M, Fieschi C, et al: Randomised double-blind placebo-controlled trial of thrombolytic therapy with intravenous alteplase in acute ischaemic stroke (ECASS II). Second European-Australasian Acute Stroke Study Investigators. Lancet 1998;352:1245-1251.

33 Hacke W, Kaste M, Bluhmki E, et al: Thrombolysis with alteplase 3-4.5 h after acute ischemic stroke. $\mathrm{N}$ Engl J Med 2008;359:1317-1329.

34 Wahlgren N, Ahmed N, Davalos A, et al: Thrombolysis with alteplase for acute ischaemic stroke in the Safe Implementation of Thrombolysis in Stroke-Monitoring Study (SITS-MOST): an observational study. Lancet 2007;369:275-282.

35 von Kummer R, Broderick JP, Campbell BC, et al: The Heidelberg Bleeding Classification: classification of bleeding events after ischemic stroke and reperfusion therapy. Stroke 2015;46:2981-2986. 